\title{
Subjectively-defined optimal/non-optimal time of day modulates controlled but not automatic retrieval processes in verbal memory
}

\author{
Delphine Puttaert ${ }^{1,2,3}$ | Stéphane Adam ${ }^{4}$ | Philippe Peigneux ${ }^{1,3}$
}

${ }^{1}$ UR2NF - Neuropsychology and Functional Neuroimaging Research Unit at CRCN, Center for Research in Cognition and Neurosciences, Université libre de Bruxelles (ULB), Brussels, Belgium

${ }^{2}$ LCFC - Laboratoire de Cartographie fonctionnelle du Cerveau, Université libre de Bruxelles (ULB), Brussels, Belgium

${ }^{3} \mathrm{UNI}$ - ULB Neurosciences Institute, Université libre de Bruxelles (ULB), Brussels, Belgium

${ }^{4}$ Psychology of Aging Unit, University of Liège (ULiege), Liège, Belgium

\section{Correspondence}

Philippe Peigneux and Delphine Puttaert, Centre for Research in Cognition \&

Neurosciences, Université Libre de Bruxelles (ULB), Bruxelles, Belgium.

Emails: philippe.peigneux@ulb.ac.be;

delphine.puttaert@ulb.ac.be

\begin{abstract}
Performance for controlled, resource-demanding retrieval in episodic memory has been consistently found to be better at an optimal compared with non-optimal time of the day, evidencing a synchrony effect. However, performance in memory tasks in which retrieval is mostly based on automatic processes was inconclusively found either to be better at a non-optimal time of day or independent of synchrony effects. A caveat in most prior studies is that optimal/non-optimal time of day is based on morningness-eveningness composite scores derived from chronotype questionnaires, which might not efficiently predict subjectively-defined cognitive efficiency periods. An additional caveat is that separate tasks are used to assess explicit and implicit retrieval in verbal memory. Indeed, no task is process-pure, and both controlled and automatic retrieval processes may potentially contribute to retrieval scores in different types of memory tasks. In the present study, we investigated the impact of individually defined subjective optimal/non-optimal time of day on verbal memory retrieval, using an adaptation of the Process-Dissociation Procedure that allows estimating the respective contributions of automatic and controlled memory retrieval processes within the same memory task. Our results disclose a higher involvement of controlled processes at subjectively optimal compared with non-optimal time of day, but no differences for automatic processes. Synchrony effects and subjectively-defined peaks and troughs of performance for controlled components of memory retrieval should be considered in the evaluation of episodic memory.
\end{abstract}

\section{KEYWORDS}

automatic processes, controlled processes, memory, Process-Dissociation Procedure, synchrony effects, time of day

\section{1 | INTRODUCTION}

The circadian rhythm, described as a nearly 24-hr endogenous oscillatory variation for sleep propensity, is known to modulate cognitive efficiency in various domains (Schmidt, Collette, Cajochen, \& Peigneux, 2007), such as sensory (Lotze, Wittmann, von Steinbüchel, Pöppel, \& Roenneberg, 1999), motor (Edwards, Waterhouse, \& Reilly, 2007), vigilance (Graw, Krauchi, Knoblauch, Wirz-Justice, \& Cajochen, 2004) and memory (Ramírez et al., 2006), with generally better performance during day than night time. Additionally, there are inter-individual differences in the phase and timing of the circadian oscillations. Morning-type chronotypes or "larks" tend to wake up early in the morning and feel the need for sleep already at early hours in the evening. Conversely, evening-type chronotypes or "owls" feel a need for sleep later in the night and wake up later in the morning. Synchrony effects, i.e. better performance at a specific time of the day for a given chronotype, were evidenced in different cognitive domains. Participants tested at their optimal moment of the day (i.e. putatively the morning for "Morning types" and the 
evening for "Evening types") exhibited better performance in executive functions (Hasher, Goldstein, \& May, 2005; May \& Hasher, 1998; Yoon, May, \& Hasher, 1999) and attentional (Correa, Molina, \& Sanabria, 2014; Natale, Alzani, \& Cicogna, 2003) tasks, as well as a reduced inclination for stereotypic judgements (Bodenhausen, 1990). Additionally, retrieval in declarative memory was found to be more efficient at an optimal compared with non-optimal time of day (Hasher, Chung, May, \& Foong, 2002; Hasher et al., 2005; May, Hasher, \& Stoltzfus, 1993; May \& Hasher, 1998; Yang, Hasher, \& Wilson, 2007). By contrast, synchrony effects are less consistent for implicit and procedural memory tasks, in which performance mostly relies on automatic retrieval processes. Indeed, some studies found a facilitating effect at non-optimal time of day for implicit memory tasks (Delpouve, Schmitz, \& Peigneux, 2014; May, Hasher, \& Foong, 2005; Rothen \& Meier, 2016), whereas others failed (Yang et al., 2007), suggesting that automatic memory retrieval processes might not, or barely, be modulated by time of day fluctuations.

Inconsistent findings may stem from caveats in previous works. First, most prior studies that tested memory functions using separate memory tasks presumed assessing the procedural and declarative components separately. However, no task is process-pure (Jacoby, 1991), and cross-contamination between explicit and implicit memory processes may not be excluded within a task. Second, the optimal and non-optimal moments for testing are most often arbitrarily defined based on a chronotype score derived from the Morningness-Eveningness Questionnaire (MEQ; Horne \& Östberg, 1976). This approach does not take into account the multi-determined (i.e. biological, cognitive and social) and inter-individual characteristics that might make a moment of the day optimal or not for cognitively demanding tasks (Dijk \& von Schantz, 2005).

In the present study, we evaluated participants at their specific optimal/non-optimal time of day, subjectively defined in terms of the maximal/minimal 2-hr periods for cognitive efficiency. We also used an adaptation (Adam, Van der Linden, Collette, Lemauvais, \& Salmon, 2005) of the Process-Dissociation Procedure (PDP; Jacoby, 1991) aimed at determining the respective contributions of controlled and automatic processes within a unique verbal memory task. We hypothesized that controlled retrieval processes in episodic verbal memory are more efficient at the optimal time of day, that is when participants are tested at a time they feel their cognitive resources are maximal, as opposed to a non-optimal time. Regarding the automatic retrieval of information, we hypothesized that it could be paradoxically more efficient at a non-optimal time of day when the efficiency of controlled processes is lowered, and thus opposes less the automatic components of memory retrieval (Delpouve et al., 2014; Schmidt et al., 2007).

\section{2 | METHODS}

\section{1 | Population and protocol}

Forty French-speaking young adults aged 20-28 years (mean age = $23.35 \pm 1.76$ years) gave their informed consent to participate in this experiment conducted in agreement with the ethical principles of the Declaration of Helsinki and approved by the Faculty Advisory Ethics committee. Exclusion criteria were excessive alcohol or psychoactive substance consumption, major neurological or psychiatric illness, extreme morning or evening chronotype (score $\leq 30$ or $\geq 70$ on the MEQ), bad sleep quality (score $>7$ on the Pittsburgh Sleep Quality Index [PSQI]; Buysse, Reynolds, Monk, Berman, \& Kupfer, 1989) and jet lag $>2 \mathrm{hr}$ in the previous month. Participants were tested within a consecutive 2 -hr period defined based on their answers to the perceived cognitive efficiency questionnaire (see below). Half of them were tested at the time of day they perceived as being optimal for their cognitive performance. The other half were evaluated at the time of day perceived as non-optimal. Participants were randomly allocated to the optimal or non-optimal experimental conditions. Participants filled in the perceived cognitive efficiency questionnaire approximately 2 weeks before their experimental session. The session included completion of questionnaires, administration of the Psychomotor Vigilance Task (PVT; Dinges \& Powell, 1985) and of the experimental PDP task (see below). The duration of the session ranged from $1 \mathrm{hr}$ to $1 \mathrm{hr} 40 \mathrm{~min}$.

\section{2 | Materials and procedure}

\subsection{1 | Questionnaires}

The PSQI (Buysse et al., 1989) assessed sleep quality and quantity for the month prior to the experiment. The St Mary's Hospital Sleep Questionnaire (Ellis et al., 1981) assessed sleep quality and quantity for the night preceding the testing day. The MEQ (Horne \& Östberg, 1976) determined the participant's chronotype. Participants also completed the Beck Depression Inventory-II (BDI-II; Beck, Steer, \& Brown, 1996) to exclude the presence of depression that may impact cognitive processes, as well as the French adaptation of the Mill-Hill Part B Vocabulary test (Deltour, 1993) considering that the experimental verbal task partly relies on the participants' vocabulary expertise.

\subsection{2 | Optimal/non-optimal subjective efficiency scale}

The subjective efficiency scale (Figure 1) aimed at determining the time of day that each participant subjectively perceived as being usually most optimal (respectively, non-optimal) for cognitive performance during a normal day (from 07:00 to 19:00 hours).

\subsection{3 | Verbal memory task and Process- Dissociation Procedure}

A detailed presentation of the verbal memory task and the theoretical framework of the PDP is provided elsewhere (Adam et al., 2005). The experimental task consisted of the continuous presentation on a computer screen of six sets of 16 words, randomly selected from a list of 96 French language bisyllabic words (e.g. "chaque"). Each word 
At which time of the day ( $2 \mathrm{~h}$ ) do you usually feel at your best and your worst to

perform on cognitive tasks?

My optimal moment:

\begin{tabular}{|c|c|c|c|c|c|c|c|c|c|c|c|c|}
\hline $7 \mathrm{~h}$ & $8 \mathrm{~h}$ & $9 \mathrm{~h}$ & $10 \mathrm{~h}$ & $11 \mathrm{~h}$ & $12 \mathrm{~h}$ & $13 \mathrm{~h}$ & $14 \mathrm{~h}$ & $15 \mathrm{~h}$ & $16 \mathrm{~h}$ & $17 \mathrm{~h}$ & $18 \mathrm{~h}$ & $19 \mathrm{~h}$ \\
\hline
\end{tabular}

My non-optimal moment:

\begin{tabular}{|l|l|l|l|l|l|l|l|l|l|l|l|l|}
\hline $7 \mathrm{~h}$ & $8 \mathrm{~h}$ & $9 \mathrm{~h}$ & $10 \mathrm{~h}$ & $11 \mathrm{~h}$ & $12 \mathrm{~h}$ & $13 \mathrm{~h}$ & $14 \mathrm{~h}$ & $15 \mathrm{~h}$ & $16 \mathrm{~h}$ & $17 \mathrm{~h}$ & $18 \mathrm{~h}$ & $19 \mathrm{~h}$ \\
\hline
\end{tabular}

FIGURE 1 Cognitive efficiency scale

was presented once, and participants were instructed to memorize it. The first syllable of the presented word (i.e. the word-stem; e.g. "cha...") was then presented after a variable lag interval (i.e. after 0 , 3 or 12 intermediate words; Figure 2). The instruction was to complete the word-stem either with the previously presented word (Inclusion condition) or with a new, different word (Exclusion condition). In the Inclusion condition, if participants could not consciously complete the stem with the previously presented word, they were instructed to complete it with the first word that came to their mind. Thus, performance in the Inclusion condition was potentially supported by controlled (with a probability C) or automatic (with a probability A) memory retrieval processes, or by a combination of both. In the Exclusion condition, participants who consciously remembered the previously presented word are able to avoid reproducing it to comply with the instruction to produce a different word (e.g. "chacal"). If, however, they generate the previously presented word in spite of the instruction, it suggests that the participant had no controlled knowledge about that word, and that her/his response was driven by the automatic trend to complete the stem with a familiar item (e.g. "cha-que"). Alternatively, inappropriate generation of a previously seen word in the Exclusion condition could be due to failed recollection and chance level. To control for this possibility, Adam et al. (2005) proposed a Baseline condition with 16 stems corresponding to words never presented in the learning list. Participants were instructed to give the first word that came to their mind. With this condition, it is possible to verify that automatic processes correspond to the unconscious influence of memory, by showing that estimates of automatic processes are significantly above the baserate or chance level to produce specific target words in response to a stem.

The 96 items were divided into six blocks of 16 items each: 16 items in the baseline condition, 16 items for Lag 0 (8 items in the Inclusion condition and 8 items in the Exclusion condition), 16 items for Lag 3 and 16 items for Lag 12 in the Inclusion condition, 16 items for Lag 3 and 16 items for Lag 12 in the Exclusion condition. Considering that the Inclusion score reflects both $\mathrm{C}$ and $\mathrm{A}$ (Inclusion $=C+A(1-C))$ influences, and the Exclusion score reflects the opposition between $C$ and $A$ (Exclusion $=A^{*}(1-C)$ ) influences, it is thus mathematically possible computing estimates of $C$ and $A$ processes and how they are modulated by subjective time of day. Condition order (Inclusion versus Exclusion) and Time of day (Optimal versus Non-Optimal) were randomly counterbalanced across participants.

\subsection{4 $\mid$ Sleepiness and vigilance}

Before the PDP task, the 10-min version of the PVT (Dinges \& Powell, 1985) was administrated. In this task, participants must press a response button as soon as a countdown clock appears on the screen, at variable intervals. Participants were also administered the

pensif / copeau / cop--- / borgne / effroi / rachat / étable / bor--- / masser / humble / étable / tenter / calque / pen---

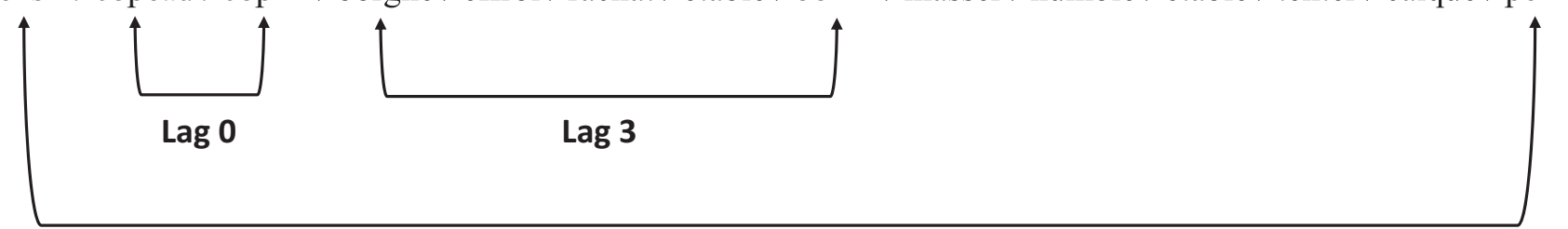

Lag 12

FIGURE 2 Experimental task. A complete word is first presented (e.g. French words "copeau", "borgne", "pensif", ...); the corresponding word-stem is then presented after a variable lag interval ranging 0 ("cop_-"), 3 ("bor_-") or 12 ("pen_-") words. Participants are instructed to complete the word-stem either with the previously presented word (Inclusion condition) or with a different one (Exclusion condition). Adapted from Adam et al. (2005) 
Karolinska Sleepiness Scale (KSS; Åkerstedt \& Gillberg, 1990) before and immediately after the experimental PDP task.

\section{3 | RESULTS}

\subsection{Questionnaires, vocabulary and time of testing}

As shown in Table 1, chronotype score was similar between participants tested at their Optimal versus Non-Optimal moment $\left(t_{38}=\right.$ $0.59, p=.558)$. The range of MEQ scores was 33-67, thus participants were neutral $(N=33)$, moderate evening $(N=4)$ or moderate morning $(N=3)$ types. Time of day of testing ranged between 08:00 hours and 18:00 hours (median 15:00 hours) in the non-optimal condition, and between 09:00 hours and 18:00 hours (median 11:00 hours) in the optimal condition. The average testing time difference between conditions was significant (Mann-Whitney U-test, $p<$ .005). Additionally, Spearman correlation analyses evidenced a negative correlation between MEQ chronotype score and Optimal moment $(r=-.375, p=.017)$, as well as a positive correlation between chronotype and Non-Optimal moment ( $r=.378, p=.016$ ).

The mean sleep duration during the previous month $\left(t_{33,451}=\right.$ 0.88, $p=.385$ ), sleep quantity (Mann-Whitney test: $U=193, p=$ $.849)$ and quality $(U=161, p=.268)$ for the previous night, vocabulary $(U=135, p=.077)$ and depression score $(U=148.5, p=.161)$ did not differ between Optimal and Non-Optimal moments. For the Mill-Hill vocabulary score, the between-group effect approached significance (0.08), but the Bayesian factor was 0.567 , i.e. inconclusive (a Bayes factor $>3$ is considered as substantial evidence for the alternative hypothesis [H1], values $<0.333$ indicate substantial evidence for the null [HO], and values between 0.333 and 3 indicate a lack of sensitivity; Dienes, 2011).

TABLE 1 Chronotype, sleep, vocabulary, depression parameters and time of testing

\begin{tabular}{lrrr|}
\hline & \multicolumn{1}{l}{ Optimal } & Non-Optimal & $p$-value \\
$\begin{array}{l}\text { MEQ } \\
\text { Global score }\end{array}$ & $50.55 \pm 8.51$ & $51.9 \pm 5.66$ & .558 \\
\hline PSQI & & & \\
\hline Global score & $5.2 \pm 2.37$ & $5 \pm 2.47$ & .989 \\
\hline Sleep duration (hr) & $8.05 \pm 0.85$ & $7.75 \pm 1.26$ & .385 \\
\hline St Mary's questionnaire & & & \\
\hline Sleep duration (hr) & $7.676 \pm 1.57$ & $7.741 \pm 1.52$ & .849 \\
\hline Quality of sleep & $4.3 \pm 0.86$ & $4.6 \pm 1.14$ & .268 \\
\hline Mill-Hill & & & \\
\hline Score (part b) & $34.95 \pm 5.07$ & $33.25 \pm 3.43$ & .077 \\
\hline BDI-Il & & & \\
\hline Score & $4.8 \pm 5.37$ & $6.25 \pm 4.39$ & .161 \\
\hline Testing time (hr) & $11.85 \pm 3.52$ & $15.05 \pm 2.89$ & .003 \\
\hline
\end{tabular}

BDI, Beck Depression Inventory-II; MEQ, Morningness-Eveningness Questionnaire; PSQI, Pittsburgh Sleep Quality Index.

\subsection{Sleepiness and vigilance}

Results disclosed higher subjective sleepiness as measured by the self-reported KSS at Non-Optimal (mean and SD: $4.3 \pm 2.11$ ) compared with Optimal (mean and SD: $3.125 \pm 1.24$; ANOVA: $F_{1,38}=$ $5.853, p=.02$ ) moments (Figure 3 ). Regarding vigilance, no momentrelated differences were found with the PVT either with performance computed on the mean reaction time $(U=195, p=.892)$ for participants tested at their Optimal (mean and SD: $341.45 \pm 23.08$ ) and Non-Optimal (mean and SD: $348.55 \pm 36.26$ ) moments, or on the reciprocal response time $(1 / \mathrm{RT})\left(t_{38}=0.768, p=.447\right)$ at Optimal (mean and SD: $3.03 \pm 0.2$ ) and Non-Optimal (mean and SD: $2.97 \pm$ 0.29) moments.

\section{3 | Word-stem completion scores}

Table 2 summarizes the proportions of stems completed with the target words for the participants evaluated at their Optimal or NonOptimal moments in the Inclusion and Exclusion (for Lag 0, Lag 3, Lag 12) and in the Baseline conditions.

In the baseline condition, an ANOVA was computed on the completion scores for the New items with the between-subject factor Moment (Optimal versus Non-Optimal) and the within-subject factor Condition (Inclusion versus Exclusion). The factors Moment $\left(F_{1,38}=\right.$ 1.134, $p=.294)$ and Condition $\left(F_{1,38}=0.01, p=.919\right)$ and their interaction were all non-significant $\left(F_{1,38}=0.507, p=.481\right)$, indicating that the probability of completing a stem with the target word by chance was the same at Optimal and Non-Optimal moments, and that both groups used the same criterion for responding in both conditions (Adam et al., 2005). Similarly, we compared completion scores at Lag 0 (i.e. immediate presentation of the stem after the word; a condition mainly ensuring that participants followed the

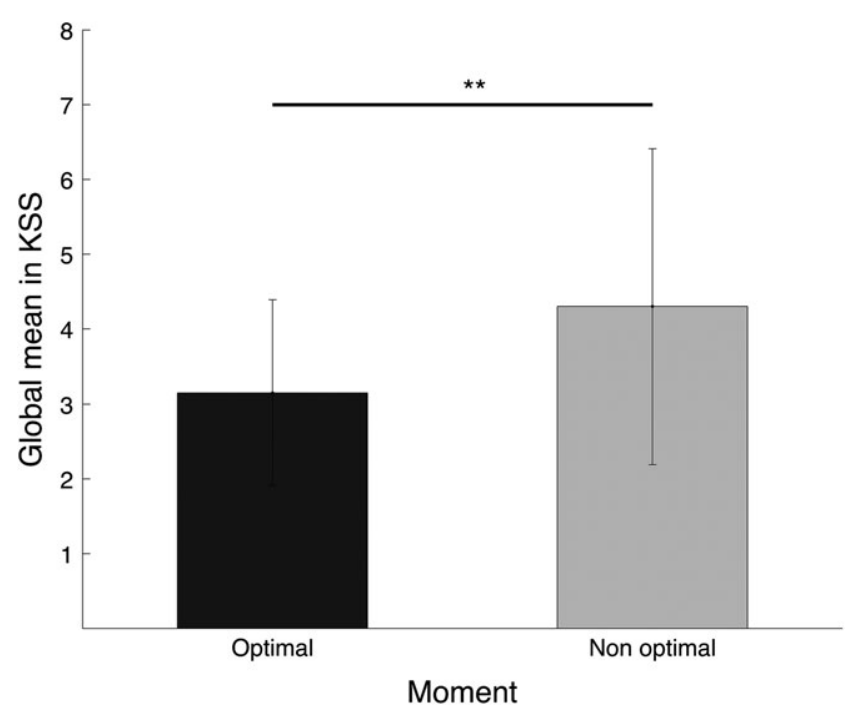

FIGURE 3 Sleepiness scores (Karolinska Sleepiness Scale; KSS) at Optimal (blue lines) versus Non-Optimal (blue squares) experimental conditions. Error bars represent standard deviations 
TABLE 2 Mean probabilities of completing stems with the target words in each condition (Inclusion and Exclusion) and for each item type (New, Lag 0, Lag 3 and Lag 12)

\begin{tabular}{|c|c|c|c|c|c|}
\hline \multirow[b]{2}{*}{ Moment } & \multirow{2}{*}{$\begin{array}{l}\text { Sample } \\
n\end{array}$} & \multicolumn{4}{|l|}{ Item type } \\
\hline & & New & Lag 0 & Lag 3 & Lag 12 \\
\hline \multicolumn{6}{|c|}{ Inclusion condition } \\
\hline Optimal & 20 & $0.118(0.069)$ & $0.987(0.03)$ & $0.84(0.11)$ & $0.759(0.09)$ \\
\hline \multicolumn{6}{|c|}{ Exclusion condition } \\
\hline Optimal & 20 & $0.106(0.06)$ & $0.006(0.027)$ & $0.071(0.08)$ & $0.1(0.09)$ \\
\hline
\end{tabular}

Standard deviations appear in parentheses. New: baseline items (never presented); Lag 0: lag interval 0 item; Lag 3: lag interval 3 items; Lag 12: lag interval 12 items.

Exclusion versus Inclusion instructions) between the Optimal and Non-Optimal conditions. No moment-related differences were found at Lag 0 both in the Inclusion $\left(t_{38}=1.453, p=.154\right)$ and Exclusion $\left(t_{38}=1, p=.324\right)$ conditions. Therefore, the remaining analyses on memory retrieval were conducted on representative Lag 3 and Lag 12 only.

Regarding completion scores for the learned items, an ANOVA was conducted on the proportion of stems completed with the target word in the Inclusion condition with the between-subject factor Moment (Optimal versus Non-Optimal) and the within-subject factor Lag interval (Lag 3 versus Lag 12). The analysis disclosed a main Lag interval effect $\left(F_{1,38}=14.807, p<.01\right)$. Post hoc planned comparisons showed that the two lag intervals significantly differed one from the other $(p<.05)$, with a decreasing completion score at increasing intervals (Lag $3>\operatorname{Lag} 12$ ). There was also a main Group effect $\left(F_{1,38}=4.192, p=.048\right)$, with a higher completion score at the Optimal (mean score and SD: $0.800 \pm 0.023$ ) compared with the Non-Optimal $(0.734 \pm 0.023)$ moment. The interaction effect was not significant $\left(F_{1,38}=0.076, p=.785\right)$.

In the Exclusion condition, a similar ANOVA conducted on the proportion of stems completed with the target word also disclosed a main Lag interval effect $\left(F_{1,38}=4.861, p=.034\right)$, with more completions (i.e. errors in this Exclusion condition) as lag interval increased. Post hoc planned comparisons evidenced differences between the two lag intervals (Lag $3<\operatorname{Lag} 12 ; p<.05$ ). There was also a main Moment effect $\left(F_{1,38}=5.828, p=.021\right)$, with a higher erroneous completion rate at the Non-Optimal $(0.164 \pm 0.023)$ compared with the Optimal $(0.086 \pm 0.023)$ moment. The Moment $\times$ Lag interval interaction effect was not significant $\left(F_{1,38}=0.778, p=.383\right)$.

\subsection{Estimates of controlled and automatic processes}

In a second step, we estimated the respective contribution of automatic and controlled memory processes (Table 3 ) by using the algebraic transformations described above (see Section 7).

The estimates of controlled processes (Figure 4) were analysed with a repeated-measures ANOVA, with the between-subject factor Moment (Optimal versus Non-Optimal) and the within-subject factor
TABLE 3 Estimates of controlled and automatic memory processes at optimal and non-optimal moments

\begin{tabular}{|c|c|c|c|}
\hline \multirow[b]{2}{*}{ Moment } & \multirow{2}{*}{$\begin{array}{l}\text { Sample } \\
n\end{array}$} & \multicolumn{2}{|c|}{ Lag intervals } \\
\hline & & Lag 3 & Lag 12 \\
\hline \multicolumn{4}{|c|}{ Controlled processes $(\mathrm{C}=\mathrm{I}-\mathrm{E})$} \\
\hline Non-optimal & 20 & $0.65(0.19)$ & $0.49(0.25)$ \\
\hline Optimal & 20 & $0.76(0.13)$ & $0.65(0.14)$ \\
\hline \multicolumn{4}{|c|}{ Automatic processes $(A=E /(1-C))$} \\
\hline Non-optimal & 20 & $0.36(0.26)$ & $0.32(0.203)$ \\
\hline Optimal & 20 & $0.31(0.29)$ & $0.25(0.21)$ \\
\hline
\end{tabular}

Standard deviations are in parentheses. Lag 3: 3 items interval; Lag 12: 12 items interval.

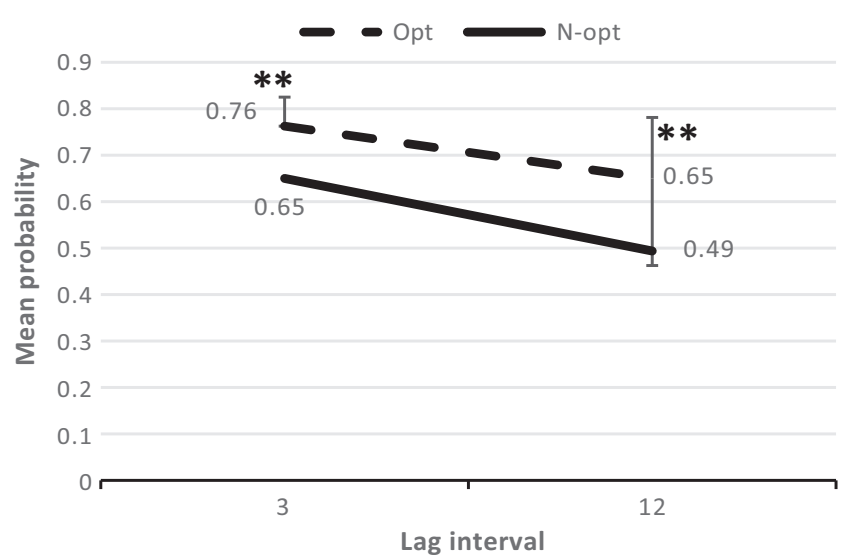

FIGURE 4 Estimates of controlled memory processes at increasing lag interval (3 versus 12) in the Optimal (Opt) and NonOptimal (N-Opt) moment conditions. ${ }^{* *} p=.01$. Error bars are standard deviations

Lag interval (Lag 3 versus Lag 12). There was a main Lag interval effect $\left(F_{1,38}=16.163, p<.001\right)$, with higher estimates of controlled processes at shorter lags (Table 3; Lag $3=0.709>$ Lag $12=0.575$; all planned comparisons $p<.05)$. There was also a main Moment effect $\left(F_{1,38}=8.533, p<.01\right)$, with higher estimates of controlled processes at the Optimal $(0.714 \pm 0.035)$ compared with the 
Non-Optimal $(0.570 \pm 0.035)$ moment. There was no interaction effect $\left(F_{1,38}=0.559, p=.459\right)$.

The estimates of automatic processes (Figure 5) were also analysed with a repeated-measures ANOVA, with the between-subject factor Moment (Optimal versus Non-Optimal) and the within-subject factor Lag interval (Lag 3 versus Lag 12). First, the analysis showed that the estimates for automatic processes were significantly higher than the probability to complete stems by chance at Lags 3 and 12 both at the Optimal moment $\left(\operatorname{Lag} 3: t_{19}=2.78, p=.012\right.$; Lag 12: $t_{19}$ $=2.93, p<.01)$ and at the Non-Optimal moment (Lag $3: t_{19}=4.11$, $p<.01$; Lag 12: $\left.t_{19}=4.57, p<.01\right)$. Second, the repeated-measures ANOVA computed on the estimates of automatic processes revealed no significant main effect of Moment $\left(F_{1,38}=0.749, p=.392\right)$ or Lag interval $\left(F_{1,38}=1.398, p=.244\right)$, nor an interaction effect $\left(F_{1,38}=\right.$ $0.064, p=.801)$

\section{DISCUSSION}

In this study, we aimed at investigating the impact of the moment of day subjectively defined as optimal or non-optimal for cognitive performance on the controlled and automatic processes subtending retrieval in episodic memory, using an adaptation (Adam et al., 2005) of the PDP (Jacoby, 1991). In line with our hypothesis and prior literature (Hasher et al., 2002; May \& Hasher, 1998; May et al., 1993; West, Murphy, Armilio, Craik, \& Stuss, 2002; Yang et al., 2007), our results disclosed more efficient controlled processes for participants evaluated at their optimal compared with their non-optimal moment. At variance, our results did not evidence any impact of the optimal versus non-optimal testing time on automatic processes in memory retrieval. This result did not confirm prior findings suggesting that automatic processes involved in implicit and procedural memory are more efficient at the individual's non-optimal moment, when controlled processes do not oppose their expression (Delpouve et al., 2014; Hasher et al., 2002; May et al., 1993, 2005).

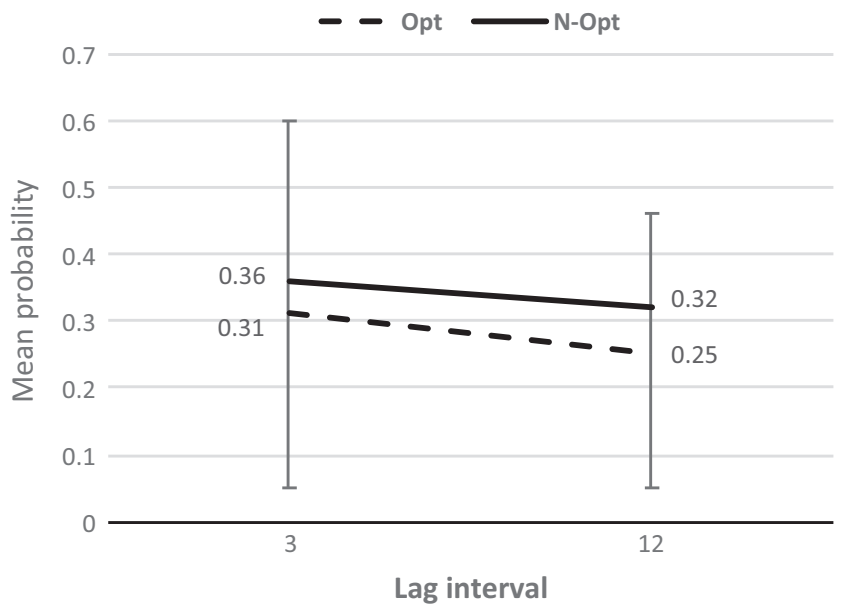

FIGURE 5 Estimates of automatic memory processes at increasing lag interval (3 versus 12) in Optimal (Opt) and NonOptima (N-Ot) moment conditions. Error bars are standard deviations
Although our results are in agreement with the findings of a previous study (Yang et al., 2007), it must be noted that their method was markedly different as they used a speeded stem completion task. Individual automatic or controlled retrieval processes were determined by comparing participants' response times in the semantic retrieval phase versus the study phase. The authors posited that controlled processes are recruited when participants slowed down in the semantic retrieval phase, whereas those who maintained stable reaction times between the semantic and study phases were assumed to have relied mostly on automatic retrieval. Although the procedure also aimed at separating controlled and automatic retrieval, increased or decreased reaction times in a memory task might also have alternative causes. For instance, changes in focused attention and vigilance can also increase reaction times in producing a correct answer. This possibility was not assessed in this previous study, unlike our current study for which vigilance and sleepiness parameters were measured. Another possible confound is that performance in a reputedly implicit memory task might be to some extent contaminated by intentional and controlled memory processes. Conversely, automatic, unintentional processes can modulate explicit memory performance. For instance, amnesiacs demonstrated evidence of knowledge on an explicit memory test when encouraged to guess (Weiskrantz \& Warrington, 1975), suggesting that memory retrieval parameters cannot be used as a process-pure measure.

In this respect, we argue that our revised version of the PDP is particularly appropriate for segregating the contribution of controlled and automatic processes within a memory task, independently of reaction time differences. The task was shown to be efficient and sensitive in pathological conditions, for instance with Alzheimer's disease (Adam et al., 2005; Knight, 1998; Smith \& Knight, 2002). Other studies used a similar PDP approach to evidence partially dissociated cerebral substrates in the acquisition of explicit and implicit knowledge in a serial reaction time task (Destrebecqz et al., 2005). Additionally, methodological precautions were taken in order to prevent problems that may limit the applicability of the PDP, such as poor task sensitivity, ceiling and/or floor effects in performance, lack of control over comprehension of instructions, and lack of baseline measurements. In the Adam et al. (2005) study, these elements were carefully accounted for in a rigorous manner, which is why we specifically chose to use this variant of the word-stem completion task to apply the PDP. Limits and boundary conditions of the PDP have also been extensively discussed in the past years (Yonelinas \& Jacoby, 2012). Nonetheless, we acknowledge that there is an ongoing debate around the different paradigms aimed at capturing controlled-automatic distinctions. For instance, the PDP and the Remember-Know (R-K) paradigms (Gardiner, Ramponi, \& RichardsonKlavehn, 1998) seem to capture partially different dimensions, which might lead to different results (Espinosa-Garcia, Vaquero, Milliken, \& Tudela, 2017). Still, Espinosa-Garcia et al. proposed that at the time of retrieval, recollection measured using the PDP might more exclusively engage controlled memory search processes than the R-K, in which case the PDP paradigm would be more appropriate for studying controlled processes in a recognition memory task. 
Discrepancies about automatic processes with prior findings might stem from our PDP method, which differs from previous studies by at least two features. First, contrary to our procedure, previous studies used separate, independent tasks to segregate controlled and automatic retrieval processes in memory (Hasher et al., 2002; May et al., 1993, 2005). For instance, Hasher et al. (2005) used two different implicit and explicit stem completion tasks, and an implicit category generation task. In the explicit task, participants were instructed to complete the stems with words of the learning list, alternatively with the first word that came to their mind. As discussed previously, conscious (controlled) and automatic recall can contribute in parallel to performance in this explicit condition, hence a contamination effect. We ourselves reported better performance at non-optimal compared with optimal time in an implicit artificial grammar learning (AGL) task (Delpouve et al., 2014), but failed in the present study to evidence differences for the automatic component of memory processing, which suggests that the effect is driven by various factors, amongst others the type of task and conditions (e.g. implicit detection of regularities during exposure to strings of letters without instruction to learn in the AGL task, whereas participants are exposed to meaningful verbal material with explicit instructions to learn for future use in the present study).

Second, synchrony effects were tested in our study by evaluating each participant at the time of day subjectively defined as being the most optimal or non-optimal moment for cognitive performance. In previous studies (Hasher et al., 2002; May \& Hasher, 1998), optimal and non-optimal time of day moments for testing were derived from the individual's chronotype score obtained after the completion of a MEQ (Horne \& Östberg, 1976). Subjective sleepiness as assessed with the KSS was higher in the non-optimal than in the optimal condition in our participants, like our prior study (Delpouve et al., 2014), suggesting the validity of a subjective determination of cognitive efficiency. Notwithstanding, further studies are needed to better understand the arguably multidimensional determinants of subjectively-defined optimal/non-optimal time of day (Dijk \& von Schantz, 2005), and as well to determine the test-retest reliability of our subjective time of day optimality measure, and to what extent it is associated with physiological modifications such as dim light melatonin onset, as shown with the well-established MEQ (Kantermann \& Eastman, 2018; Kantermann, Sung, \& Burgess, 2015). Besides differences in chronotype, preferred time of day for a demanding cognitive activity (i.e. optimal time in the context of our study) might also be subtended by various variables, such as light-dark and social cycles, meal time, environmental settings such as light exposure and motivational variables, to name a few. We argue that prior studies that used the MEQ to determine morning versus evening preference may have to some extent neglected the relative contribution of these various elements. In this respect, asking participants to define optimal versus non-optimal time of day purely based on their subjective feeling is a possible way to circumvent the difficulty to control these variables. Interestingly, we disclosed moderate negative and positive correlations between the MEQ score and subjectively-defined Optimal and Non-Optimal moments, respectively, suggesting that MEQ actually captures elements related to optimal and non-optimal time of day for cognitive performance. Conversely, subjective determination of optimal and non-optimal periods in an individual partially reflects chronotype, with the additional advantage that a purely subjective evaluation is more precise in the determination of the best/ worst moment within a day to carry on a specific activity, if we consider the wide range of hours defined as optimal (09:00-18:00 hours) or non-optimal (08:00-18:00 hours) by our participants. Nonetheless, it was recently reported that young neutral type individuals (like in the present study) do not exhibit significant effects of time of testing over the day (i.e. morning, midday and evening) on inhibitory processing, flexibility, memory, perceptual speed and access to well-learned knowledge (May \& Hasher, 2017). Although the results from this latter study might suggest limited variations in cognitive performance at the group level all over the day for neutral chronotype participants exhibiting similar features than our population, it is also possible that assessments made at three fixed time of day periods do not capture the subjective experience of optimality/ non-optimality at the individual level, which we have seen widely distributed all along the day across participants. From our perspective, it reinforces the hypothesis that time of day for high versus poor cognitive performance might be better defined using subjectively-defined periods. If our hypothesis is correct, differences in performance between subjectively-defined optimal and non-optimal time of day should be evidenced for the same tasks than the ones used in the May and Hasher (2017) study. Further studies should investigate this issue.

A potential limitation of our study is that we used a betweensubject design (i.e. participants were tested either at their optimal or non-optimal moment) due to task constraints, whereas a within-subject design might have better controlled for inter-individual differences. Also, the impact of other cognitive processes on the controlled and automatic components of memory retrieval should be accounted for and specifically tested in future studies. For instance, reduced inhibition at non-optimal time of day can also impact correct response production, especially in the Exclusion condition in which perseverations must be avoided. Likewise, changes in cognitive flexibility may also interfere with performance when participants need to switch response patterns between the Inclusion and Exclusion conditions.

Altogether, our results emphasize the importance of accounting for potential interactions between controlled and automatic memory processes in the neuropsychological evaluation of episodic verbal memory, in addition to the time of day at which tasks are completed.

\section{ACKNOWLEDGEMENTS}

The experimental study was conducted at the Université Libre de Bruxelles (ULB). DP is currently a PhD student supported by a grant from the Fonds Erasme Foundation (ULB, Belgium). The authors thank the participants for their cooperation, and Jeromy Hrabovecky for editing the manuscript for English language. 


\section{CONFLICT OF INTERESTS}

The authors are not aware of any affiliation, funding or financial holdings that might be perceived as affecting the objectivity of this paper. The authors declare that there is no biomedical financial interest or potential conflict of interest.

\section{AUTHOR CONTRIBUTIONS}

DP and PP designed the study; DP, SA and PP wrote the protocol; DP collected the data, DP and PP conducted the analyses. All authors interpreted the data and contributed to the writing. All authors have approved the final manuscript.

\section{REFERENCES}

Adam, S., Van der Linden, M., Collette, F., Lemauvais, L., \& Salmon, E. (2005). Further exploration of controlled and automatic memory processes in early Alzheimer's disease. Neuropsychology, 19, 420-427. https://doi.org/10.1037/0894-4105.19.4.420

Åkerstedt, T., \& Gillberg, M. (1990). Subjective and objective sleepiness in the active individual. International Journal of Neuroscience, 52, 2937. https://doi.org/10.3109/00207459008994241

Beck, A. T., Steer, R. A., \& Brown, G. K. (1996). Manual for the Beck depression inventory-II(pp. 1-82). San Antonio: TX Psychol.

Bodenhausen, G. V. (1990). Stereotypes as judgmental heuristics: Evidence of circadian variations in discrimination. Psychological Science, 1, 319-322. https://doi.org/10.1111/j.1467-9280.1990.tb00226.x

Buysse, D. J., Reynolds, C. F., Monk, T. H., Berman, S. R., \& Kupfer, D. J. (1989). The Pittsburgh Sleep Quality Index: A new instrument for psychiatric practice and research. Psychiatry Research, 28, 193-213. https://doi.org/10.1016/0165-1781(89)90047-4

Correa, Á., Molina, E., \& Sanabria, D. (2014). Effects of chronotype and time of day on the vigilance decrement during simulated driving. Accident Analysis and Prevention, 67, 113-118. https://doi.org/10. 1016/j.aap.2014.02.020

Delpouve, J., Schmitz, R., \& Peigneux, P. (2014). Implicit learning is better at subjectively defined non-optimal time of day. Cortex, 58, 18-22. https://doi.org/10.1016/j.cortex.2014.05.006

Deltour, J. J.; Echelle de vocabulaire de Mill Hill de JC Raven. (1993). Adaptation française et normes européennes du Mill Hill et du Standard Progressive Matrices de Raven (PM38). Braine-le-Château, Belgium: L'Application des Techniques Modernes.

Destrebecqz, A., Peigneux, P., Laureys, S., Degueldre, C., Del Fiore, G., Aerts, J., ... Maquet, P. (2005). The neural correlates of implicit and explicit sequence learning: Interacting networks revealed by the process dissociation procedure. Learning \& Memory, 12, 480-490. https://doi.org/10.1101/Im.95605

Dienes, Z. (2011). Bayesian versus orthodox statistics: Which side are you on? Perspectives on Psychological Science, 6, 274-290. https://doi. org/10.1177/1745691611406920

Dijk, D.-J., \& von Schantz, M. (2005). Timing and consolidation of human sleep, wakefulness, and performance by a symphony of oscillators. Journal of Biological Rhythms, 20, 279-290. https://doi.org/10.1177/ 0748730405278292

Dinges, D. F., \& Powell, J. W. (1985). Microcomputer analyses of performance on a portable, simple visual RT task during sustained operations. Behavior Research Methods, Instruments, \& Computers, 17, 652655. https://doi.org/10.3758/BF03200977

Edwards, B., Waterhouse, J., \& Reilly, T. (2007). The effects of circadian rhythmicity and time-awake on a simple motor task. Chronobiology
International, 24, 1109-1124. https://doi.org/10.1080/07420520701 795316

Ellis, B. W., Johns, M. W., Lancaster, R., Raptopoulos, P., Angelopoulos, N., \& Priest, R. G. (1981). The St. Mary's Hospital sleep questionnaire: A study of reliability. Sleep, 9, 3-97.

Espinosa-Garcia, M., Vaquero, J. M., Milliken, B., \& Tudela, P. (2017). Recollection and familiarity for words and faces: A study comparing Remember-Know judgments and the Process Dissociation Procedure. Memory, 25, 19-34. https://doi.org/10.1080/09658211.2015. 1120310

Gardiner, J. M., Ramponi, C., \& Richardson-Klavehn, A. (1998). Experiences of remembering, knowing and guessing. Consciousness and Cognition, 7, 1-26. https://doi.org/10.1006/ccog.1997.0321

Graw, P., Krauchi, K., Knoblauch, V., Wirz-Justice, A., \& Cajochen, C. (2004). Circadian and wake-dependent modulation of fastest and slowest reaction times during the psychomotor vigilance task. Physiology \& Behavior, 80, 695-701. https://doi.org/10.1016/j.physbeh. 2003.12.004

Hasher, L., Chung, C., May, C. P., \& Foong, N. (2002). Age, time of testing, and proactive interference. Canadian Journal of Experimental Psychology, 56, 200-207. https://doi.org/10.1037/h0087397

Hasher, L., Goldstein, D., \& May, C. P. (2005). It's about time: Circadian rhythms, memory, and aging. In C. Izawa \& N. Ohta (Eds.), Human learning and memory: Advances in theory and application: The 4th Tsukuba International Conference on Memory (pp. 199-217). Mahwah, NJ: Lawrence Erlbaum Associates Publishers.

Horne, J. A., \& Östberg, O. (1976). A self-assessment questionnaire to determine morningness-eveningness in human circadian rhythms. International Journal of Chronobiology, 2, 97-110.

Jacoby, L. L. (1991). A process dissociation framework: Separating automatic from intentional uses of memory. Journal of Memory and Language, 30, 513-541. https://doi.org/10.1016/0749-596X(91)90025-F

Kantermann, T., \& Eastman, C. I. (2018). Circadian phase, circadian period and chronotype are reproducible over months. Chronobiology International, 35, 280-288. https://doi.org/10.1080/07420528.2017.1400979

Kantermann, T., Sung, H., \& Burgess, H. J. (2015). Comparing the morningness-eveningness questionnaire and Munich chronotype questionnaire to the dim light melatonin onset. Journal of Biological Rhythms, 30, 449-453. https://doi.org/10.1177/0748730415597520

Knight, R. G. (1998). Controlled and automatic memory process in Alzheimer's disease. Cortex, 34, 427-435. https://doi.org/10.1016/S00109452(08)70765-2

Lotze, M., Wittmann, M., von Steinbüchel, N., Pöppel, E., \& Roenneberg, T. (1999). Daily rhythm of temporal resolution in the auditory system. Cortex, 35, 89-100. https://doi.org/10.1016/S0010-9452(08)70787-1

May, C. P., \& Hasher, L. (1998). Synchrony effects in inhibitory control over thought and action. Journal of Experimental Psychology: Human Perception and Performance, 24, 363-379.

May, C. P., \& Hasher, L. (2017). Synchrony affects performance for older but not younger neutral-type adults. Timing \& Time Perception, 5, 129-148. https://doi.org/10.1163/22134468-00002087

May, C. P., Hasher, L., \& Foong, N. (2005). Implicit memory, age, and time of day: Paradoxical priming effects. Psychological Science, 16, 96-100. https://doi.org/10.1111/j.0956-7976.2005.00788.x

May, C. P., Hasher, L., \& Stoltzfus, E. R. (1993). Optimal time of day and the magnitude of age differences in memory. Psychological Science, 4 326-330. https://doi.org/10.1111/j.1467-9280.1993.tb00573.x

Natale, V., Alzani, A., \& Cicogna, P. C. (2003). Cognitive efficiency and circadian typologies: A diurnal study. Personality and Individual Differences, 35, 1089-1105. https://doi.org/10.1016/S0191-8869(02) 00320-3

Ramírez, C., Talamantes, J., García, A., Morales, M., Valdez, P., \& MennaBarreto, L. (2006). Circadian rhythms in phonological and visuospatial storage components of working memory. Biological Rhythm Research, 37, 433-441. https://doi.org/10.1080/09291010600870404 
Rothen, N., \& Meier, B. (2016). Time of day affects implicit memory for unattended stimuli. Consciousness and Cognition, 46, 1-6. https://doi. org/10.1016/j.concog.2016.09.012

Schmidt, C., Collette, F., Cajochen, C., \& Peigneux, P. (2007). A time to think: Circadian rhythms in human cognition. Cognitive Neuropsychology, 24, 755-789. https://doi.org/10.1080/02643290701754158

Smith, J. A., \& Knight, R. G. (2002). Memory processing in Alzheimer's disease. Neuropsychologia, 40, 666-682. https://doi.org/10.1016/ S0028-3932(01)00137-3

Weiskrantz, L., \& Warrington, E. K. (1975). The problem of the amnesic syndrome in man and animal. The Hippocampus, 2, 283-291.

West, R., Murphy, K. J., Armilio, M. L., Craik, F. I. M., \& Stuss, D. T. (2002). Effects of time of day on age differences in working memory. Journals of Gerontology. Series B, Psychological Sciences and Social Sciences, 57, 3-10. https://doi.org/10.1093/geronb/57.1.P3

Yang, L., Hasher, L., \& Wilson, D. E. (2007). Synchrony effects in automatic and controlled retrieval. Psychonomic Bulletin \& Review, 14, 51-56. https://doi.org/10.3758/BF03194027
Yonelinas, A. P., \& Jacoby, L. L. (2012). The process-dissociation approach two decades later: Convergence, boundary conditions, and new directions. Memory \& Cognition, 40, 663-680. https://doi.org/10.3758/ s13421-012-0205-5

Yoon, C., May, C. P., \& Hasher, L. (1999). Aging, circadian arousal patterns and cognition. In N. Schwarz, D. Park, B. Knauper \& S. Sudman (Eds.), Aging, cognition and self reports (pp. 117-143). Washington, DC: Psychological Press.

How to cite this article: Puttaert D, Adam S, Peigneux P. Subjectively-defined optimal/non-optimal time of day modulates controlled but not automatic retrieval processes in verbal memory. J Sleep Res. 2018;e12798. https://doi.org/ $10.1111 /$ jsr.12798 